\title{
Cyclodehydration Reaction of Polyhydrazides. III. Influence of the Sample History
}

\author{
B. GEBBEN, E. ROLEVINK, M. H. V. MULDER, and \\ C. A. SMOLDERS, University of Twente, Department of Chemical \\ Technology, P.O. Box 217, 7500 AE Enschede, The Netherlands
}

\begin{abstract}
Synopsis
The influence of preparation history upon the thermal cyclodehydration reaction of polyhydrazide samples has been investigated. Solid polyhydrazide samples were prepared from DMSO solutions using the phase-inversion technique. Significant differences in conversion rates were observed between samples prepared by nonsolvent immersion precipitation and by evaporation of solvent. It appeared that contact with the nonsolvent water during the sample preparation process has considerable influence on the conversion rate. Not only does the immersion in and washing with water lead to a better removal of the solvent also the $\mathrm{pH}$ of the water determines the rate of conversion. A distinct change in the conversion rate is found for washing water at a $\mathrm{pH}$ of 7 . Basic washing solutions showing lower conversion rates than acid ones. A mechanism explaining these phenomena is proposed.
\end{abstract}

\section{INTRODUCTION}

In two previous papers ${ }^{1,2}$ the kinetics of thermal cyclodehydration reaction of polyhydrazide in the solid state has been studied using both nonisothermal and isothermal thermogravimetry. One of the results from these studies was the dependence of the reaction kinetics on the history of the polyhydrazide sample. A striking difference was observed between the kinetics of the powder, being the washed and dried polycondensation product, and a homogeneous film prepared with the same polymer. The film, prepared from the powder by solution casting and evaporation of the solvent, showed considerably higher conversion rates than the original powder. From the kinetics determined for the cyclodehydration reaction it was previously concluded that the ratedetermining step in the whole process was an internal rotation of chain segments towards more favorable chain conformations for the ring closure reaction. The expected reaction scheme for a hydrazide group is presented in Figure 1.

The transition of the extended form a to the folded form $b$, being rotational isomers, is proposed to be the rate determining step, unless the initial conformation is already in the folded form. However, the folded conformation is believed to be less favorable because of the expected oxygen repulsion. Many different conformations are possible by rotation of the hydrazide group round the $\mathbf{N}-\mathbf{N}$ axis, form $\mathbf{a}$ and $b$ being the extreme conformations. The transition from structure $\mathbf{b}$ to $\mathbf{c}$, being the keto-enol tautomers, is suggested by Korshak. ${ }^{4}$ This transition is believed to be necessary for the final dehydra- 


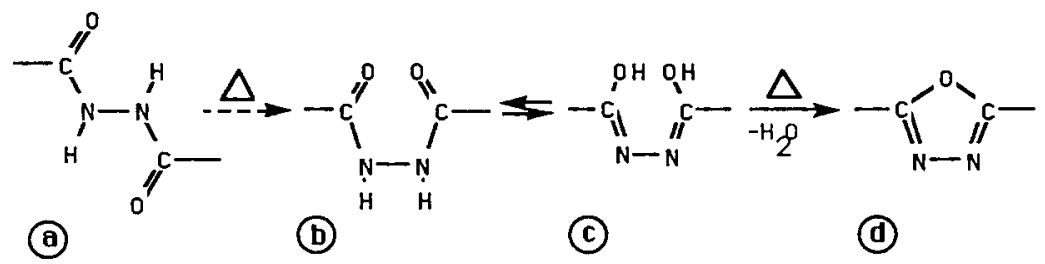

Fig. 1. The expected reaction scheme for the thermal cyclodehydration reaction of the hydrazide group.

tion reaction because this reaction probably proceeds through a transition state close to the structure of the enol form (form c).

In the context of the rate-determining $\mathbf{a}$ to $\mathbf{b}$ transition the observed difference in kinetics between powder and film was explained in terms of a difference in initial chain conformation. Both Frazer ${ }^{3}$ and Korshak ${ }^{4}$ noticed a considerable influence of orientation upon the subsequent conversion rate of their polyhydrazide fibers. The extended conformation (isomer a) is believed to be present preferentially in the more oriented (drawn) fibers, causing a slower conversion rate of these fibers compared to the less oriented fibers. These observations therefore strongly support the proposed reaction mechanism.

A possible difference in initial chain conformation between powder and film should be related with the process by which the solid polymer samples are prepared. Both samples are prepared from solution using a phase-inversion technique. Several factors within this process can be determining, e.g., the composition of the starting polymer solution, possible orientation during shaping and/or solidification, the kind of phase-inversion process, the use of a nonsolvent or not, the kind of nonsolvent, and the rate at which the phaseinversion process proceeds.

In this paper the determining factor or factors in the sample preparation process will be elucidated using isothermal thermogravimetry on polymer samples prepared in different ways. For comparison of the kinetics isothermal conversions at a temperature of $290^{\circ} \mathrm{C}$ over a period of $1 \mathrm{~h}$ were followed. The polyhydrazide used for these experiments was poly-(1,3-phenyl-1,4-phenyl)hydrazide (TIPH) as in the previous papers. The results are used to further unravel the conversion mechanism.

\section{EXPERIMENTAL}

\section{Materials}

Materials and polymer preparation were described in a previous paper. ${ }^{1}$ Three new batches of poly-(1,3-phenyl-1,4-phenyl)-hydrazide (TIPH), TIPH1, -2 , and -3 , respectively, were used for the experiments described in this paper. (TIPH1: $\eta_{\text {inh }}=1.16 \mathrm{dL} / \mathrm{g}$, TIPH2: $\eta_{\text {inh }}=0.92 \mathrm{dL} / \mathrm{g}$, and TIPH3: $\eta_{\text {inh }}=1.00$ $\mathrm{dL} / \mathrm{g})$

Water of a high purity was obtained by hyperfiltration of demineralized water using hollosep membranes (Toyobo). Methanol (analysis grade), was obtained by Merck and used as received. 


\section{Sample Preparation}

The original powder samples were prepared by pouring the polycondensation reaction mixture (NMP with $\mathrm{LiCl}$ ) in a large excess of water under continuous stirring after which the precipitated polymer was washed several times with water and dried.

Films were prepared from a $6-15$ wt\% solution of the polymer in DMSO by casting the solution on a glass plate using a casting knife with a slit-height of $0.20 \mathrm{~mm}$. Homogeneous films were obtained by evaporation of the solvent in a $\mathrm{N}_{2}$-atmosphere at room temperature for at least $12 \mathrm{~h}$ followed by an additional drying procedure at $150^{\circ} \mathrm{C}$ and reduced pressure for $16 \mathrm{~h}$. Asymmetric, porous membranes were obtained by immersing the casted films in a water bath at room temperature, after which the films received the same washing treatment as the powder samples.

Additional washing treatments were performed by soaking the samples in a large excess of the washing medium for 2 days. The $\mathrm{pH}$ value of the washing water was measured using a glass electrode at the moment before the polymer sample was removed from the water.

\section{Thermogravimetry}

A Perkin-Elmer TGS-2 Thermogravimetric Analyser in combination with a system 4 Microprocessor Controller and a Thermal analysis Data Station Model 3700 was used for the isothermal thermogravimetry. During these experiments a nitrogen atmosphere was provided by a continusous flow of nitrogen gas through the sample holder of $85 \mathrm{~mL} / \mathrm{min}$. All samples were held isothermally at $200^{\circ} \mathrm{C}$ for $20 \mathrm{~min}$ to remove adsorbed water till a constant weight was reached. Then the sample was heated with a heating rate of $200^{\circ} \mathrm{C} / \mathrm{min}$ to $290^{\circ} \mathrm{C}$ and kept at that temperature. The actual value, measured with a thermocouple near the sample was $290.3 \pm 0.2^{\circ} \mathrm{C}$ for all samples. The point $t=0$ was taken at the moment where the first weight loss was observed. From measurements of the exact temperature value near the samples a time interval of about $2 \mathrm{~min}$ was observed after $t=0$ before the temperature reached its final constant value.

For calculation of the degree of conversion from the thermogravimetric data the theoretical value for the maximum weight loss was used, analogous to the procedure in previous papers, being $11.0 \%$ of the initial dry weight which was calculated from the structural unit formula taking into account the nonreactive endgroups. Some of the samples were heated at $290^{\circ} \mathrm{C}$ over an extended period of 2 days to determine the experimental maximal weight loss and to calculate the final degree of conversion.

\section{NMR Spectroscopy}

A Nicolet NT200, $200 \mathrm{MHz}$ FT-NMR spectrometer was used for obtaining the ${ }^{1} \mathrm{H}$-NMR spectra of TIPH solutions in deuterated DMSO (99.96\%).

\section{RESULTS AND DISCUSSION}

Figure 2 shows $1 \mathrm{~h}$ isotherms at $290^{\circ} \mathrm{C}$ for several samples of TIPH1 prepared in different ways. All samples were prepared from the same DMSO 


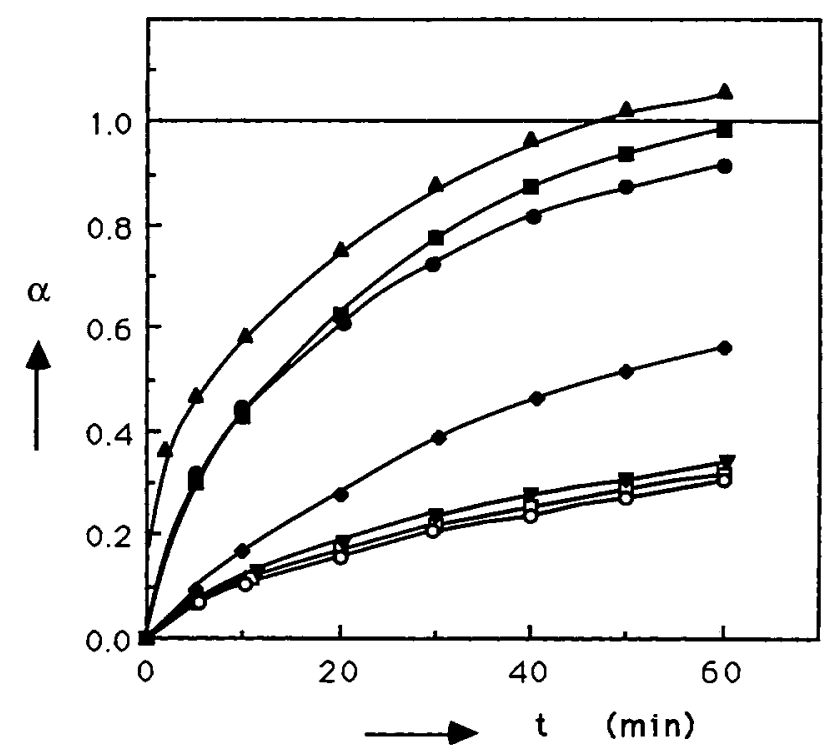

Fig. 2. Isotherms for TIPH1 samples prepared from the same DMSO solution: homogeneous film casted ( $\Lambda$ ), homogeneous film poured $(\square)$, homogeneous film washed $(\uparrow)$ and powder $(O)$; from the same DMSO solution now containing $\mathrm{LiCl}$ : homogeneous film (๑), homogeneous film washed ( $\nabla)$, and powder ( $\square$ ).

solution but with different post-treatments. Some of the solutions also contained $\mathrm{LiCl}$ to check the influence of this salt, because $\mathrm{LiCl}$ is always present in the polycondensation reaction mixture from which the original powders are prepared. Some of the films were obtained by pouring instead of casting the solution on the glass plate in order to prevent possible orientation of the polymer molecules by casting. To investigate the influence of washing some of the films prepared by evaporation were also extensively washed afterwards, just as it is done with the powders.

In agreement with the results presented in the previous papers this figure again shows the distinct difference in conversion rate between the powders prepared by immersion precipitation and the films prepared by evaporation. The composition of the polymer solution does not seem to be an important factor as samples prepared from exactly the same polymer solution still show considerable differences. The addition of $\mathrm{LiCl}$ to the DMSO solution hardly has any influence on the kinetics and there is also no significant difference in conversion rate between films casted or poured on the glass plate. However, if the films are given the same washing treatment as is the case with the powders the conversion rates clearly decrease towards the rates normally found for the powders. From this set of varied parameters the contact with the nonsolvent seems to be a decisive factor for the conversion rate.

For an explanation of these differences in the observed kinetics the extent of solvent removal comes to mind as a first possibility. The nonwashed film possibly contains some residual solvent because it was observed that the final degrees of conversion of the films exceed the theoretical maximum of 1.0, while the other samples approach this value fairly well. An incorrect degree of 


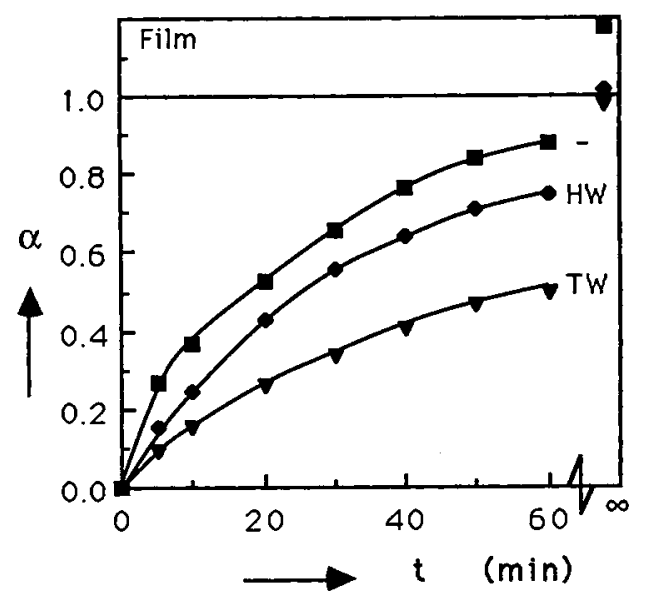

Fig. 3. Isotherms for TIPH2 homogeneous films before (ש) and after washing with tap water $(\mathrm{TW})(\nabla)$ or hyperfiltrated water $(\mathrm{HW})(\diamond)$.

conversion will result from the additional weight loss of solvent. Furthermore traces of residual solvent present in the polymer matrix may act as plasticizer and enhance the mobility of the chain segments, thus influencing the conversion kinetics. The influence of washing and the nature of the nonsolvent used for washing treatments is further examined. In Figure 3 the isotherms for a homogeneous film of TIPH2 are presented before and after additional washing with tap water or with hyperfiltrated water. In this figure also the final degrees of conversion are presented. In agreement with Figure 2, it can be seen that as a result of washing a decrease in conversion rate and a better approach of the final theoretical value for conversion is observed, probably indicating a better removal of residual solvent.

Looking at the shape of the isotherms it seems that loss of residual solvent takes place at the very beginning of the process. This is in good agreement with the observed lower energies of activation found for the process in its early stage in the previous two papers. Additional evidence for the presence and removal of residual DMSO in the nonwashed films can be obtained by sulfur analysis of a TIPH1 film before $(2.2 \pm 0.2 \% \mathrm{~S})$ and after washing $(0.3 \pm 0.2 \% \mathrm{~S})$. A reduction in sulfur content of about $90 \%$ can be observed after washing.

Above this effect of solvent removal also the nature of the nonsolvent used for the washing treatment seems to influence the rate of conversion. The use of tap water clearly results in much lower conversion rates than the use of hyperfiltrated water. Figure 4 presents the isotherms for powders prepared from a DMSO solution by nonsolvent precipitation in three different nonsolvents (tap water, hyperfiltrated water, and methanol), followed by washing with the same medium. Again it seems that the nature of the nonsolvent, used for precipitation and washing, influences the conversion rate. The conversion of the original powders can be accelerated using hyperfiltrated water or methanol instead of tap water.

An even better illustration of the influence of the nature of the nonsolvent can be observed in the preparation of asymmetric porous membranes which 


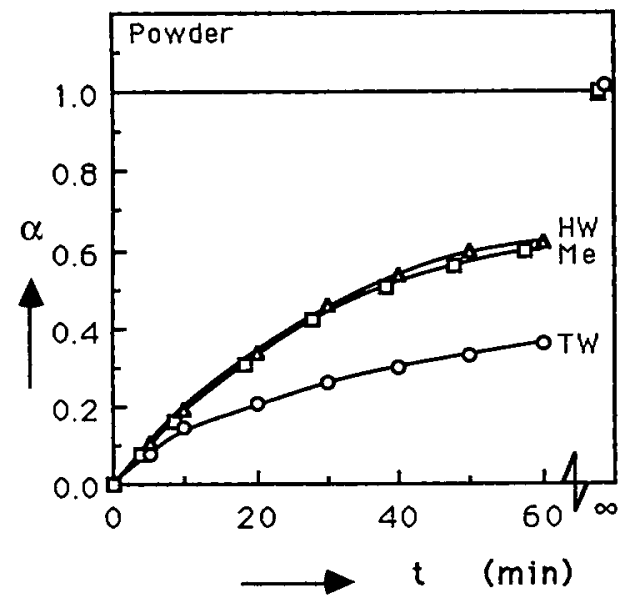

Fig. 4. Isotherms for TIPH1 powder prepared from a $6 \%$ solution in DMSO by non-solvent precipitation and washing in tap water (TW) (O), in hyperfiltrated water (HW) $(\Delta)$, or in methanol (Me) (口).

are made by casting the polymer solution on a glass plate (comparable to the homogeneous film preparation) followed by immersion precipitation in a nonsolvent (comparable to the powder preparation). These isotherms are presented in Figure 5 showing that the use of tap water during immersion precipitation results in asymmetric membranes with conversion rates comparable to the rates found for the original powder (also prepared using tap water), while the use of hyperfiltrated water leads to conversion rates approaching the rates found for the evaporated film.

All washed samples finally reach their theoretical weight loss indicating that washing with all three media has been effective. A difference in washing power between the three nonsolvents therefore may not be expected. Besides a

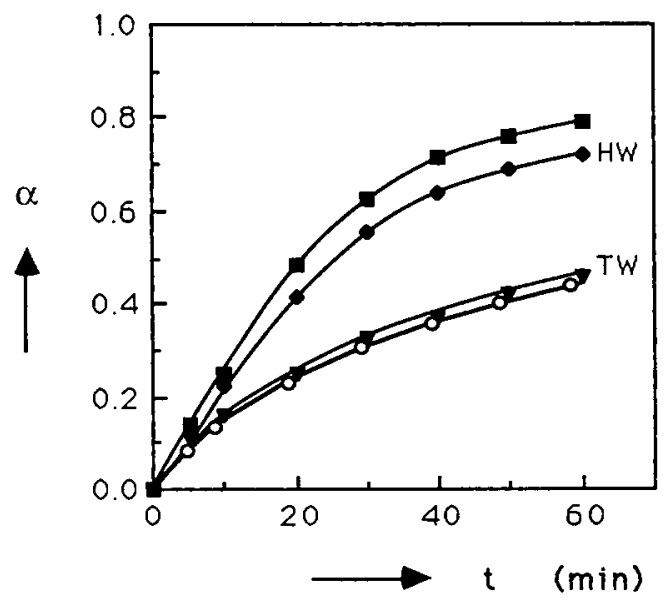

Fig. 5. Isotherms for immersion precipitated TIPH2 films in tap water (TW) ( $\nabla$ ) and

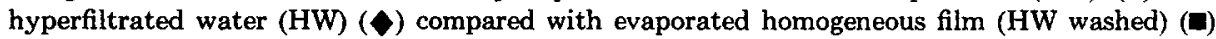
and the original TIPH2 powder $(\mathrm{O})$. 


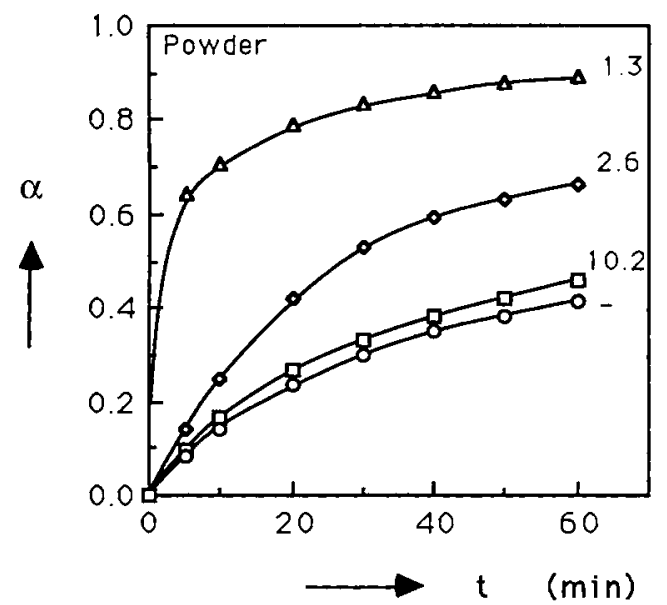

Fig. 6. Isotherms for TIPH3 powder, before $(O)$ and after additional washing with hyperfiltrated water given different $\mathrm{pH}$ values, $1.3(\Delta), 2.6(\diamond)$, and $10.2(\square)$.

better removal of residual solvent by washing these experiments have also indicated an influence of the nature of the nonsolvent upon the rate of conversion. The difference between tap water and hyperfiltrated water is especially striking. Contact with tap water in all cases leads to considerably lower conversion rates than with other media.

One of the differences between tap water and hyperfiltrated water is the presence of low molecular weight substances and ions in the former. Another point is a small difference in the $\mathrm{pH}$ value, the tap water in our laboratories being slightly more basic $(\mathrm{pH}=7.5-8.0)$ compared to the hyperfiltrated water used $(\mathrm{pH}=6.7)$. In order to test the influence of the $\mathrm{pH}$ of the water, film and powder samples of TIPH3 were given an additional washing treatment with hyperfiltrated water, to avoid the presence of ions, which was given different $\mathrm{pH}$ values by addition of sulfuric acid or sodium hydroxide. From Figures 6 and 7, showing the isotherms of the washed films and powders respectively, a clear $\mathrm{pH}$ influence can be observed: higher conversion rates are obtained for $\mathrm{pH}$ values lower than 7 , while lower conversion rates are obtained after washing at $\mathrm{pH}$ higher than 7 .

The conversion rate for the powder can be accelerated and that for the film can be slowed down by controlling the $\mathrm{pH}$ of the water. Water with a $\mathrm{pH}$ value of 1.3 does result in an enormous increase in conversion rate for the powder. It has become so fast that already during the fast heating up towards the isothermal temperature of $290^{\circ} \mathrm{C}$ there is a considerable amount of conversion. Basic water on the contrary does not seem to decrease the rate in comparison with that for to the original powder.

Looking more extensively at the $\mathrm{pH}$ influence, Figure 8 shows the relation between the observed degree of conversion obtained after $1 \mathrm{~h}$ at $290^{\circ} \mathrm{C}$ for TIPH3 powders and the $\mathrm{pH}$ value of the washing water. An interesting step-wise pattern can be observed. A distinct change in the conversion kinetics can be observed around a $\mathrm{pH}$ value of 7 : above $\mathrm{pH}=7$ a constant low degree of conversion is obtained comparable to the original powder values and below 


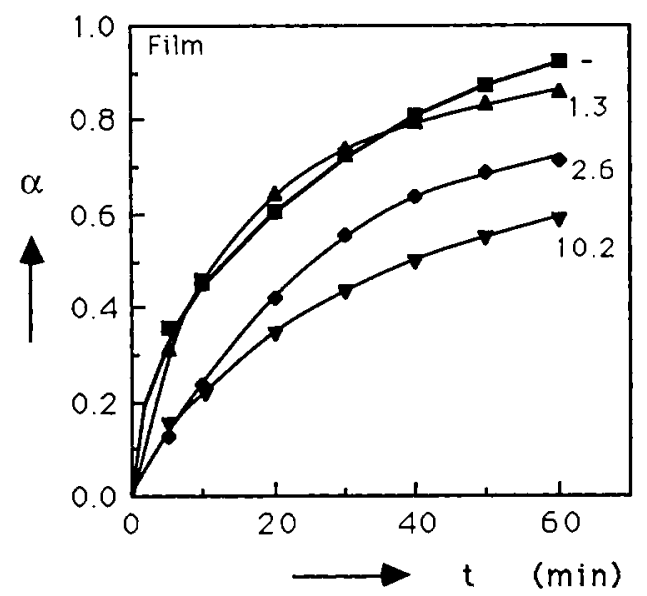

Fig. 7. Isotherms for TIPH3 homogeneous films, before (更) and after additional washing with hyperfiltrated water given different $\mathrm{pH}$ values, $1.3(\wedge), 2.6(\bullet)$, and $10.2(\nabla)$.

$\mathrm{pH}=7$ a constant but higher degree of conversion is observed. The same trend in $\mathrm{pH}$ dependency can be observed when tap water is used instead of hyperfiltrated water for the above presented washing treatments. Below $\mathrm{pH}=2$ increasingly higher degrees of conversion are found but it was noticed that in this acidic region, below $\mathrm{pH}=2$, the maximal experimental weight loss exceeds the theoretical value markedly and simultaneous degradation has to be feared. Viscosity measurements have shown that the inherent viscosity of powders washed with water of a $\mathrm{pH}$ value below 2 has decreased considerably while the inherent viscosity remains unchanged after washing above $\mathrm{pH}=2$.

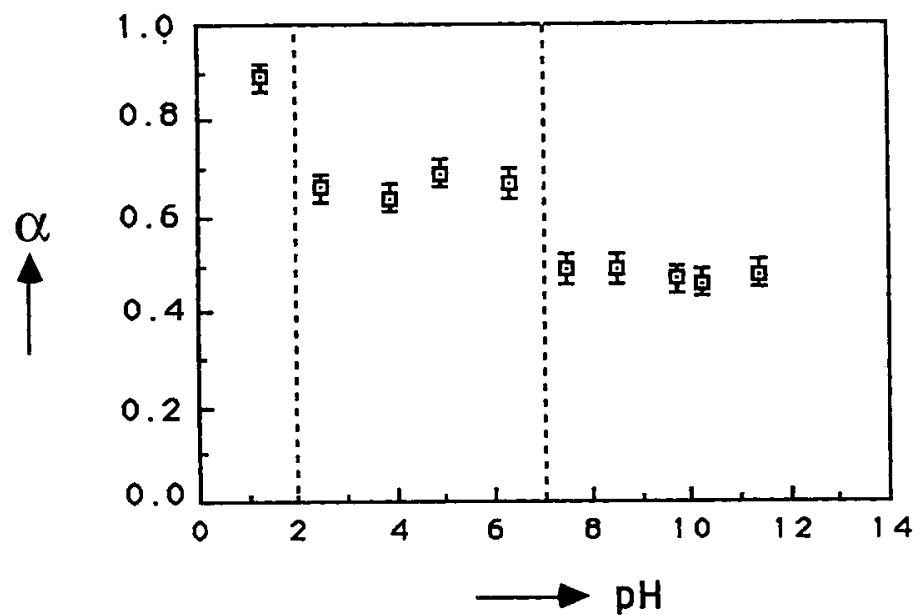

Fig. 8. The degree of conversion reached after $1 \mathrm{~h}$ at $290^{\circ} \mathrm{C}$ for TIPH3 powder as a function of the $\mathrm{pH}$ of the washing water. 
Apart from an effect on the conversion rate the $\mathrm{pH}$ also has an effect on the color of the dried polymer sample: from yellow after washing at high $\mathrm{pH}$ values to white after washing at low $\mathrm{pH}$ values. It is believed that polyhydrazides are tautomeric and that the hydrazide group can exist in the enol and the keto form. Frazer ${ }^{5}$ reported that in an acidic environment the hydrazide groups are expected to be preferentially in the keto form (form a and $\mathbf{b}$ in Figure 1), resulting in a white colored polymer, while in a basic environment the enol form (form c) is more favorable, resulting in a yellow colored polymer. He gave no further evidence of the coexistence of the tautomers.

When the dehydration reaction proceeds through the enol form of the hydrazide group, as some authors believe, the enol form would be expected to be the more favorable one for the conversion reaction. ${ }^{4,5}$ Nevertheless, the contrary is observed; in the above described experiments there is an inhibition of the reaction when the polymer has been in a basic environment. However, this can be explained in terms of chain mobility.

Figure 9 shows the possible keto-enol tautomerization equilibria for a polyhydrazide segment, where all the hydrazide groups are in the extended conformation. Except for the extreme all-ketonic form (I) and the all-enolic form (III), Figure 9 also shows an intermediate form where one hydrazide group contains both a keto and an enol form of the amid group (II). This is a likely conformation because of the possibility of stabilization by an internal hydrogen bond between the hydroxyl group and the carbonyl oxygen of the same hydrazide group. Besides this keto-enol tautomerism, Figure 9 also shows the possibility for the enol form to have a resonance possibility (III + IV) by which the enol form is stabilized. For TIPH this form IV however is less stable than form III because of the charge separation. It can be seen that on going from form I to III, this means shifting the equilibrium to the enol side, the conjugation of the system increases and the axes of free internal rotation, the $-\mathrm{N}-\mathrm{N}-$, the $-\mathrm{C}-\mathrm{N}-$, and $-\mathrm{C}-\mathrm{C}-$ bonds, become more and more blocked for rotation. For hydrazide groups in the extended conformation, as is shown in Figure 9, rotation towards the more favorable folded form will be more difficult when these hydrazide groups are mainly in the enol form.

The position of these equilibria is expected to be very sensitive to the polymer environment. A solvent dependency of the keto/enol fraction has been observed for other tautomers in literature., ${ }^{6,7}$ The equilibrium seems to depend on the polarity, the hydrogen bond donor and acceptor capacity of the solvents, as well as on the concentration of the solute. In addition to these factors, one might expect the possibility for intermolecular hydrogen bonding between neighboring chains, as well as the possibility for intramolecular hydrogen bonding to be factors influencing the keto-enol equilibrium. In this way the chain conformation can influence the position of the keto-enol equilibrium and perhaps vice versa.

The coupling of this keto-enol equilibrium with the mobility/flexibility of the polyhydrazide chains may be the explanation for the observed environmental influence in the case of the conversion of polyhydrazides. Apparently the equilibrium position of this keto-enol transition is rather sensitive to 


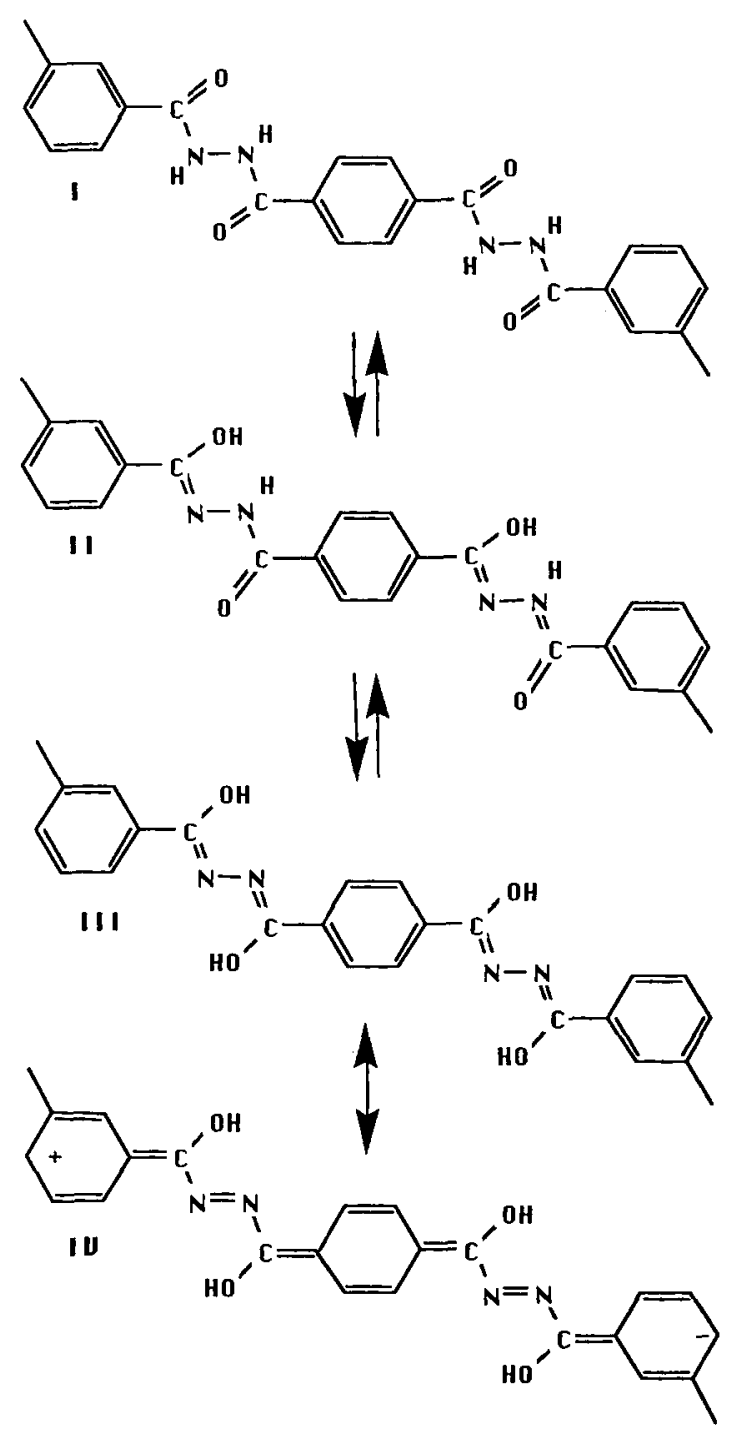

Fig. 9. Schematic representation of the keto-enol tautomerism for an extended TIPH chain segment.

acidic or basic conditions around $\mathrm{pH}=7$. Therefore the observed difference in effect on conversion between tap water $(\mathrm{pH}=7.6)$ and hyperfiltrated water $(\mathrm{pH}=6.7)$ might be caused by a subtle difference in $\mathrm{pH}$ value.

It is known from literature that certain anions such as $\mathrm{Li}^{+}$and $\mathrm{Ca}^{2+}$ can interact with peptide groups ${ }^{8,9}$ and, more specifically, Frazer found that polyhydrazides are known to form chelates at high concentrations of metal ions such as $\mathrm{Cu}^{2+}, \mathrm{Co}^{2+}$, and others. ${ }^{10}$ Both types of interactions are schematically shown in Figure 10. Deliberately introducing metal ions $\left(\mathrm{Cu}^{2+}\right.$ and $\left.\mathrm{Li}^{+}\right)$ to hyperfiltrated water did not have a noticeable influence on the conversion rate except that in agreement with a statement by Frazer ${ }^{9}$ a simultaneous 

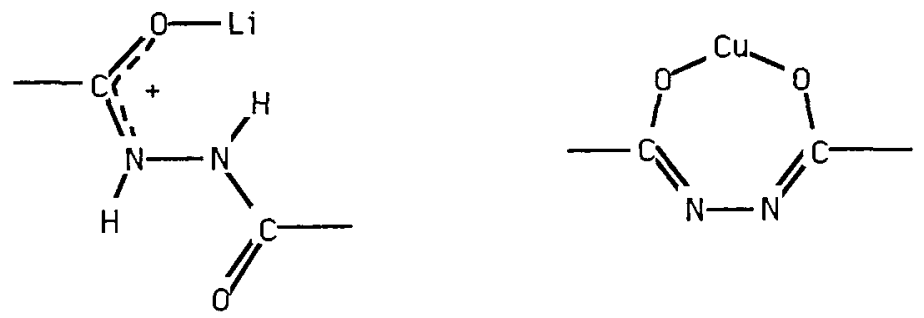

Fig. 10. Schematic representation of possible cation interactions with the hydrazide group.

degradation during conversion upon the addition of copper ions was observed. The direct influence of cations on the chemical structure and the consequences for the conversion rate remain unknown and have not been examined further.

It is known from work of Frazer ${ }^{10}$ that the thermal cyclization reaction of polyhydrazides in the solid state is catalyzed by acids. He tested several acids and observed that the strength of the catalyst was dependent on the $\mathrm{pK}_{a}$ of the acid, from which he concluded that protonation of the hydrazide group was a major step in the catalysis mechanism. He did not include chain mobility in his explanation for the catalytic effect of acids. We think that the above proposed keto-enol transition is a significant factor for the chain mobility and that making use of this fact can help to explain the acid catalysis qualitatively.

Films of polyhydrazide are prepared from solutions in DMSO, which is an aprotic polar solvent. There is absolutely no way in which the hydrazide groups could be protonated when prepared from DMSO but still the reaction proceeds much faster than for the powders. In this case it is certainly not a protonation which makes the conversion fast but possibly the polarity of the surrounding medium of the polymer which contributes to its initial configuration and chain mobility. It is clearly shown in Figures 2 and 4 that the conversion for films can be slowed down by washing with tap water, giving a reduction which can not be assigned only to removal of solvent. A considerable change in conformation of the solid polyhydrazide sample upon contact with water is not very likely as this would imply a considerable movement of the chains. A shift in the keto-enol equilibrium of the polymer from the keto to the enol side of this equilibrium therefore seems to be the best explanation. The extent of this shift being dependent on the $\mathrm{pH}$ value of the water used.

Apart from the visible color difference $(\mathrm{pH}<7$ : white or colorless and $\mathrm{pH}>7$ : yellow), it is difficult to evidence the existence of the keto and enol form. Infrared analysis using colorless and yellow homogeneous films (acid and base washed, respectively) showed no difference in spectra. Both films showed equally strong and broad carbonyl stretching peaks at $1650 \mathrm{~cm}^{-1}$. Other analyses were performed with dilute solutions in DMSO. Ultraviolet spectra could not be made properly using DMSO solutions because DMSO itself shows considerable absorbance in the ultraviolet range. In the visible range however the differences are obvious because of the color difference. The yellow solution showed an absorption at $\lambda_{\max }=390 \mathrm{~nm}$. Also ${ }^{1} \mathrm{H}-\mathrm{NMR}$ spectra (presented in Figure 11) are identical although the dilute DMSO- $d_{6}$ solutions were markedly different in color. 

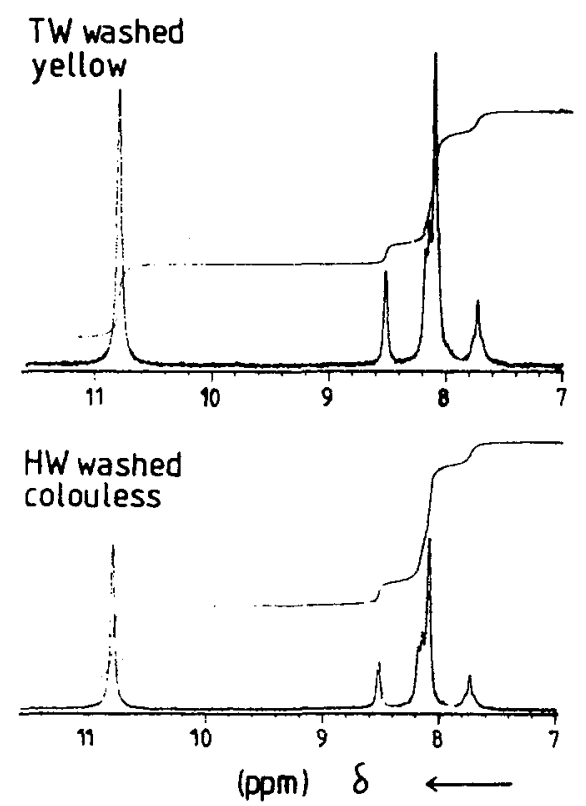

Fig. 11. ${ }^{1}$ H-NMR spectra in DMSO- $d_{6}$ of TIPH3, washed with tap water (TW) to give a yellow colored polymer solution and with hyperfiltrated water (HW) to give a colorless solution.

The ${ }^{1} \mathrm{H}$-NMR spectrum does give us some clue concerning the conformation of the hydrazide groups in solution. The signal at $\delta=8.13 \mathrm{ppm}$ is assigned to the aromatic protons, the other signal at $\delta=10.77 \mathrm{ppm}$ must represent the hydrazide protons. The fact that only one singlet is observed indicates that these protons belong either to an extreme side of the keto-enol equilibrium or the average position of the transition which is possible when the transition is much faster than the time constant of the NMR measurement. Apart from this the considerable shift of these protons indicates acidic protons or a structure where the protons are involved in hydrogen bonds. The color difference can be explained by a difference in degree of conjugation. In Figure 12 the most probable conformation is presented, an extended conformation with internal hydrogen bonds. This conformation allows for a very fast keto-enol transition, the presence of internal hydrogen bonds, and a difference in degree of conjugation.

The foregoing evidence for the extended conformation of course represents the conformation in dilute solution. It is not allowed to extend this evidence to conformations existing in the solid state where also intermolecular hydro-

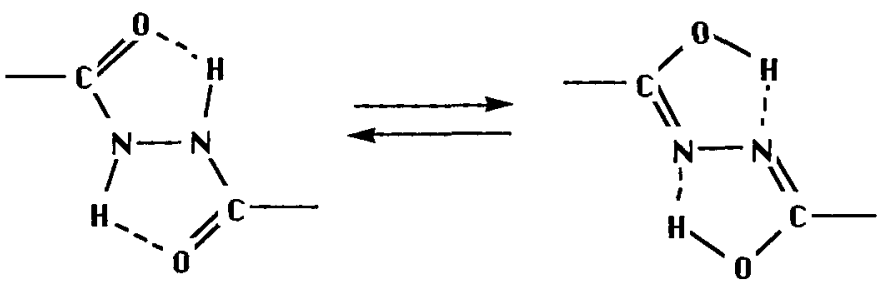

Fig. 12. Proposed hydrazide conformation in solution according to NMR interpretation. 
gen bonds will influence the type of conformation and where quite different structures can be expected. It does give us an indication that these conformations are possible.

The experiments described in this paper have shown that the cyclodehydration reaction kinetics of a polyhydrazide is very responsive to its history. The effect of polymer orientation was already known from the literature. ${ }^{4,5}$ Orientation probably changes the conformation of the chains, it will increase the extended to folded ratio. Now it has been shown that also the nature of the environment can influence the kinetics considerably, probably by an effect on the tautomerism of the hydrazide groups. It is this phenomenon which is believed to contribute largely to the differences between powders and films observed in the previous two papers.

\section{CONCLUSIONS}

The history dependence observed in the previous papers of the thermal cyclodehydration reaction of polyhydrazide in the solid state is caused by the phase inversion process. If the polymer sample has been prepared by nonsolvent precipitation and has been in contact with tap water, the rate of conversion is much lower compared to polymers prepared by evaporation of solvent. Besides a more complete removal of solvent by immersion precipitation an additional influence of the nature of the nonsolvent is observed. Asymmetric membranes prepared by nonsolvent precipitation showed much higher rates of conversion when hyperfiltrated water was used instead of tap water. The $\mathrm{pH}$ of the water used has a considerable influence. A distinct change in conversion rate is observed at a $\mathrm{pH}$ value of about 7. Below $\mathrm{pH}=7$ higher rates of conversion have been observed. This $\mathrm{pH}$ dependency is explained by the sensitivity of the keto-enol tautomerization equilibrium of the hydrazide groups for the environment and the effect of the tautomeric forms and their degree of conjugation on chain mobility. Apart from a possible difference in initial chain conformation also a difference in the position of the keto-enol equilibrium of the hydrazide groups determines the conversion rate of solid polyhydrazides into polyoxadiazoles.

\section{References}

1. B. Gebben, M. H. V. Mulder, and C. A. Smolders, J. Polym. Sci. Polym. Chem. Ed., 26, 1743-1755 (1988).

2. B. Gebben, M. H. V. Mulder, and C. A. Smolders, J. Polym. Sci. Polym. Chem. Ed., 26, $1757-1768$ (1988).

3. A. H. Frazer and F. T. Wallenberger, J. Polym. Sci. A, 2, 1137-1156 (1964).

4. V. V. Korshak, Vysokomol. Soyed. A, 16(8), 1745-1722 (1974) (English translation).

5. A. H. Frazer, W. Sweeny, and F. T. Wallenberger, J. Polym. Sci. A, 2, 1157-1169 (1964).

6. S. Masusa, M. Tanaka, and T. Ota, Makromol. Chem., 187, 1087-1095 (1986).

7. S. Masusa, M. Tanaka, and T. Ota, J. Polym. Sci. Polym. Lett. Ed., 24, 529-532 (1986).

8. D. Balasubramanian and R. Shaikh, Biopolymers, 12, 1639-1650 (1973).

9. D. Balasubramanian and B. C. Misra, Biopolymers, 14, 1019-1026 (1975).

10. A. H. Frazer and F. T. Wallenberger, J. Polym. Sci. A, 2, 1825-1832 (1964).

11. A. H. Frazer and D. R. Wilson, J. Appl. Polym. Sci. Appl. Polym. Symp., 9 (1969).

12. A. H. Frazer and W. P. Fitzgerald Jr., J. Polym. Sci. C, 19, 95-101 (1967).

Received July 11,1988

Accepted January 17, 1989 